\title{
Electron Temperature Fluctuations on the Eve and after the NEIAL Events Observed with the EISCAT Svalbard Radar
}

\author{
B. DAlipi ${ }^{a}$, N. Syla ${ }^{b}$ AND F. Aliaj ${ }^{b, *}$ \\ ${ }^{a}$ University "Kadri Zeka", Faculty of Education, Zija Shemsiu Str. 3, 60000 Gjilan, Kosovo \\ ${ }^{b}$ University of Prishtina, Department of Physics, Eqrem Cabej Str. 51, 10000 Prishtina, Kosovo
}

\begin{abstract}
This work presents studies of naturally enhanced ion acoustic lines and the correlation they have with the electron temperature fluctuations on the eve and after the NEIAL events. The methodology used in this work is based on the analysis of European Incoherent Scatter Scientific Association raw data, archived at RutherfordAppleton Laboratory (Harwell Oxford Campus) in the United Kingdom. The EISCAT Svalbard radar raw data were analyzed using two specialized programs: the Real Time Graph and the Grand Unified Incoherent Scatter Data Analysis Program, version 8.6. Our findings suggest a strong correlation between the NEIALs phenomenon and plasma parameter fluctuations such as the electron temperature in the Earth ionosphere. Our findings show that the plasma electron temperature progressively increases before the event, and progressively decreases after the event.
\end{abstract}

DOI: 10.12693/APhysPolA.137.509

PACS/topics: space plasma physics, ionospheric physics, radar science, plasma diagnostics, electron temperature

\section{Introduction}

Robinson et al. [1] emphasized a relationship between the electron temperature and auroral emissions. They pointed out that auroral emissions are associated with electron temperatures that reach $6000 \mathrm{~K}$. Blixt et al. [2] found a strong relationship between NEIALs and dynamic rayed aurora, as well as red background emissions. Rietveld et al. [3] reported that the electron temperatures at high latitude ionosphere varied between $4000 \mathrm{~K}$ and $8000 \mathrm{~K}$ at $450 \mathrm{~km}$ and that the ratio $\mathrm{Te} / \mathrm{Ti} \geq 2$ was a prerequisite for the occurrence of NEIALs.

In their articles, Foster et al. [3] and Wahlund et al. [4] stated that NEIALs are associated with high electron temperatures. Moreover, Wahlund et al. [5] observed NEIALs events together with topside ionospheric electron temperature enhancement and a good correlation between enhancements in both electron density and electron temperature. Forme et al. [6] and Forme and Fontaine [7] found that increased electron temperatures are often associated with strongly asymmetric enhanced ion lines, namely NEIALs. Lunde et al. [8] suggested that high-energy particles may have an important part to play in the generation of NEIALs.

An incoherent scatter radar technique is capable of producing a spectral panel as an output that represents the physical conditions of the ionospheric plasma and its properties. A very important parameter that can be deduced using this technique is the electron temperature. There is a close relationship between the shape and the

*corresponding author; e-mail: fisnik.aliaj@uni-pr.edu size of the spectra and the ionospheric plasma parameters. Some theoretical examples of the ion line shapes and the sizes, corresponding to the power scattered by different ionospheric ion species, are shown in Ref. [9].

\section{Material and methods}

Our target data, used to observe the NEIAL events, were produced during the run of EISCAT Svalbard IS Radar at Longyearbyen, placed at about $78^{\circ} \mathrm{N}$ and $16^{\circ} \mathrm{E}$ (geographical) on June 1, 2004. The radar antenna was $42 \mathrm{~m}$ in diameter, fixed, field-aligned, and with an emitting/receiving capability. The working frequency for this radar was $500 \mathrm{MHz}$

The data was subject to analysis with two specialized programs, Real Time Graph (RTG) and Grand Unified Incoherent Scatter Data Analysis Program (GUISDAP, version 8.6) [10]. GUISDAP enabled us to deduce and analyze the ionospheric parameters, while RTG (ver. 2.6) enabled us to monitor and to observe, analytically, the NEIALs spectrum. The experiment used was tau0 (6.4 second version) and the scan pattern was Common Program CP. The cover of the altitude height started from $77.9 \mathrm{~km}$ and reached up to $1253.2 \mathrm{~km}$ above sea surface.

The target observation day was June 1, 2004. We have observed successively, one by one, every single data dump spectrum and have evaluated their spectral morphology. Some chosen data dumps, those that were in the vicinity of data dumps containing a NEIAL signature, were analyzed with GUISDAP to calculate electron temperature values. It is worth mentioning that during the time of observation, no heating experiment was conducted, i.e. the ionosphere over the Svalbard was not artificially modified. The results were purely naturally influenced. 
As it is known, we cannot believe the GUISDAP analysis outcomes that come from NEIAL events. For those non-Maxwellian events, the analyses may produce some results, but those results do not tell us the right answer about the plasma parameters. Therefore, we will use an approach to make our argument about the behaviour of electron temperature during a NEIALs event.

\subsection{The approach definition}

We are able to analyse plasma parameters before and after the NEIAL event because the plasma parameters are in a Maxwellian state and the results produced using GUISDAP analysis are believable. Otherwise, the data dumps containing NEIAL signatures are not analysable by means of the standard method, using GUISDAP, because the plasma parameters are in a non-Maxwellian state. Therefore, if we can notice that before the NEIAL event the electron temperature increases systematically and on the contrary it decreases systematically after the NEIAL event, this might be an argument to conclude that such events are associated with an increase of electron temperature.

\subsection{Procedure in short}

First, we have identified the radar data dumps, around the time the NEIAL has happened. The data dumps showing normal spectral morphology have been analysed, while we have rejected analyzing every profile showing abnormal spectral morphology or NEIALs event. The result outcomes, in terms of electron temperature, were used in our analysis according to the approach defined above. The numerical results were used to analyse the behaviour of the electron temperature during naturally enhanced ion-acoustic lines events at high latitude ionosphere, using the above mentioned approach, by applying the regression analysis among the temperature values. The regression was applied for every altitude gate that corresponded with NEIAL signature heights, as observed in corresponding spectrum panels.

\section{Results and discussion}

The most pronounced NEIAL event during our observation routine (June 1, 2004) lies in the interval between 07:35:56 UT and 07:36:16 UT. The maximum of the power returned was integrated in the third data dump and was equal to $19000 \mathrm{~K}$ [11].

Figure 1 shows two classes of spectrum morphologies that differ substantially. Figure 1b shows the spectrum morphology obtained after the integration of the whole NEIAL event, contained in 9 consecutive data dumps (07:35:56 UT to 07:36:48 UT). Figure 1a represents the spectrum morphology of six integrated data dumps, immediately prior to the event, while Fig. 1c represents the spectrum morphology of six integrated data dumps immediately after the event.
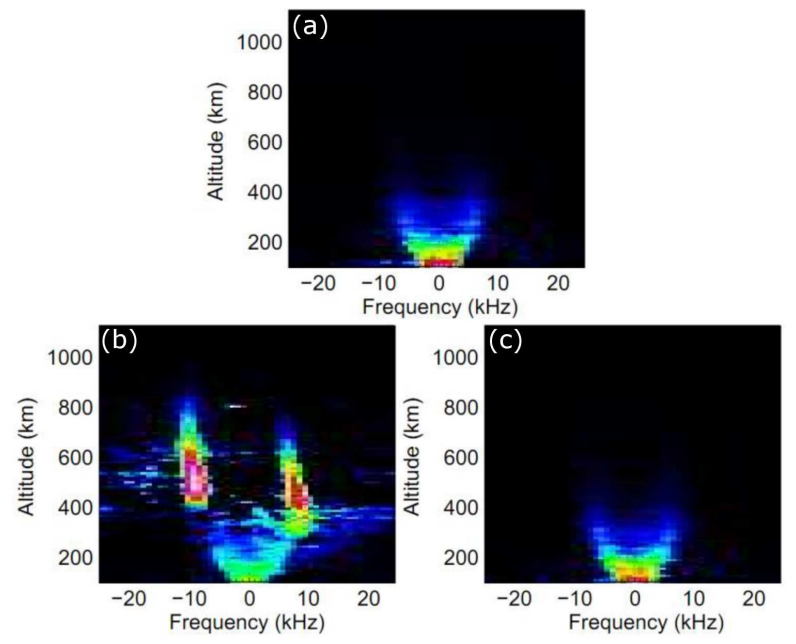

Fig. 1. (a) Immediately prior to the event, (b) integration during the NEIAL event, (c) immediately after the event.
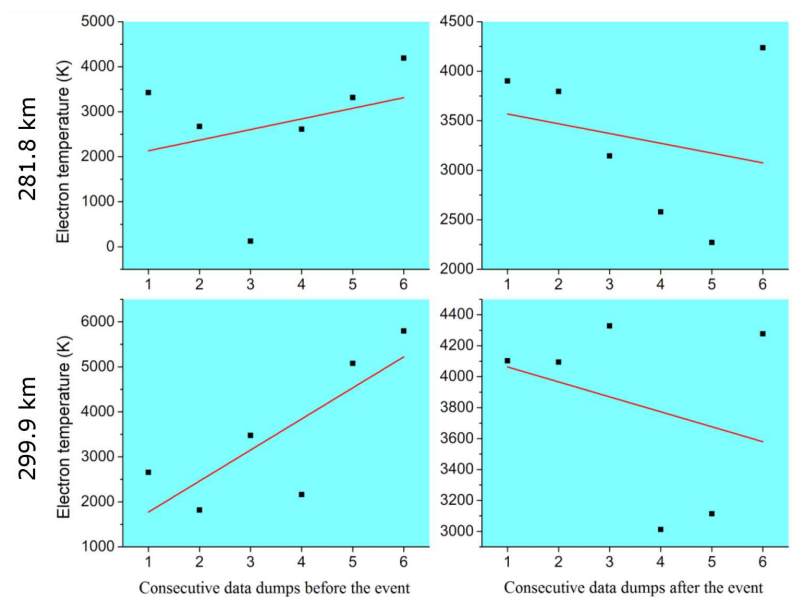

Fig. 2. An example of linear regression for electron temperature values for first two latitude gates (gate 24 and gate 25). The left parts represent regression lines of electron temperature for data dumps before the event and the right parts represent regression lines of electron temperature for data dumps after the event.

Based on a visual inspection of the spectral morphology for the selected event, we found that the altitude profile corresponding with altitude gates, from 24 up to 37, were contaminated with NEIALs.

Applying a GUISDAP analysis to our selected data dumps, we were able to derive the target data for our analysis, using a linear regression method. Figure 2 shows an example of linear regression for electron temperature values for first two altitude gates.

Our analysis shows that in all gates, namely starting at gate $24(281.8 \mathrm{~km})$ up to the gate $37(451.75 \mathrm{~km})$, the coefficient of slope before the event had a positive value. Based on these results, we can surely conclude that there was a systematic increase of temperature immediately 


\section{TABLE I}

The regression coefficient and the intercept for temperature values of successive data dumps obtained with GUISDAP. Analysis includes 14 gates that correspond to the altitude gates covered by a NEIAL event, viz. gate 24 $(281.8 \mathrm{~km})$ to gate $37(613.8 \mathrm{~km})$. Slope - regression coefficient (before and after the event), Temp. -intercept of the regression line with $y$ coordinate, namely the electron temperature (before and after the NEIAL event).

\begin{tabular}{c|c|c|c|c}
\hline \hline \multirow{2}{*}{ Gate } & \multicolumn{2}{|c|}{ Slope } & \multicolumn{2}{c}{ Temp. [K] } \\
\cline { 2 - 5 } & before & after & before & after \\
\hline gate 24 & 235.84 & -98.8 & 1898 & 3667 \\
gate 25 & 690.28 & -96.69 & 1080 & 4157 \\
gate 26 & 266.28 & -3382.87 & 2405 & 21482 \\
gate 27 & 131.51 & 95.72 & 2597 & 5015 \\
gate 28 & 317.34 & -85.77 & 2429 & 4053 \\
gate 29 & 191.35 & -69.97 & 2902 & 4330 \\
gate 30 & 254.95 & 63.52 & 2930 & 3981 \\
gate 31 & 88.43 & -285.23 & 4709 & 5280 \\
gate 32 & 38.3 & -409.85 & 5032 & 5882 \\
gate 33 & 165.89 & -478.99 & 3556 & 7542 \\
gate 34 & 644.71 & -177.55 & 2343 & 5802 \\
gate 35 & 335.48 & -899.22 & 4565 & 5890 \\
gate 36 & 202.03 & -401.42 & 4522 & 6558 \\
gate 37 & 451.75 & -667.132 & 2340 & 8355 \\
\hline average & 286.72 & -492.44 & 3093 & 6692
\end{tabular}

prior to the event. On the other hand, the analysis for the same altitude gates after the event, showed that twelve gates had a negative coefficient of slope. Applying these results to our given approach, we can conclude that there was a systematic decrease of the temperature values immediately after the NEIAL event.

The above conclusion can be supported by the data in Table I. The average of the regression coefficient, before NEIAL event was +286.72 , while that after the event was -492.44. Therefore, it is obvious that before the event electron temperatures were increasing and on the contrary they were decreasing after the event. This is a strong argument to state that before the NEIAL events, plasma is associated with an increase of electron temperatures.

Further, we have analysed the intercept of the regression line with the temperature axle for every target gate, before and after the event. The results are shown in Table I. The average temperature for considered gates was around $3092 \mathrm{~K}$, while after the event the average temperature resolved around $6692 \mathrm{~K}$.

The difference between the values of temperature was very obvious (Figs. 3 and 4). The temperature values on average doubled after the event. These findings give us an argument to conclude that the ionospheric plasma processes, manifesting NEIALs, were associated with an increase of plasma electron temperature. Our findings about the electron temperature behavior during NEIAL events agree with Rietveld et al. [3].

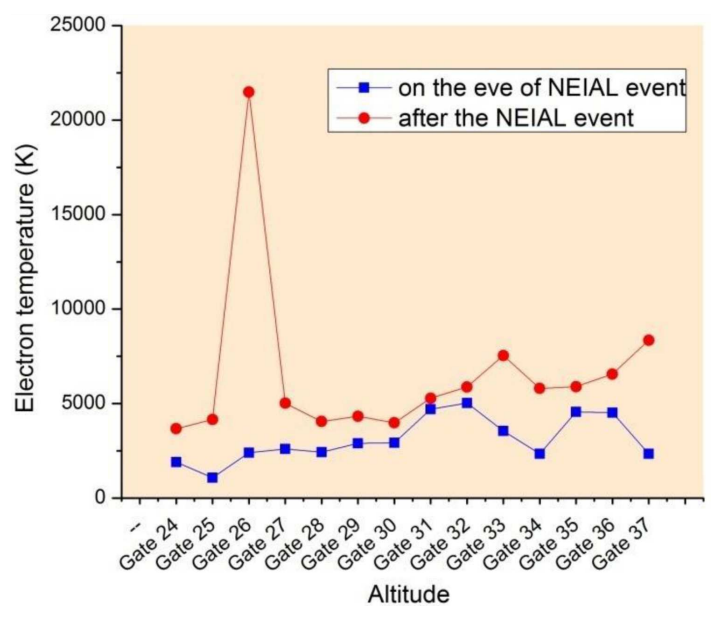

Fig. 3. Comparison of the electron temperature on the eve (the red line plot) and after the NEIAL event (the blue line plot), for every studied altitude gate in the interval between 07:35:56 UT and 07:36:16 UT (June 1, 2004).

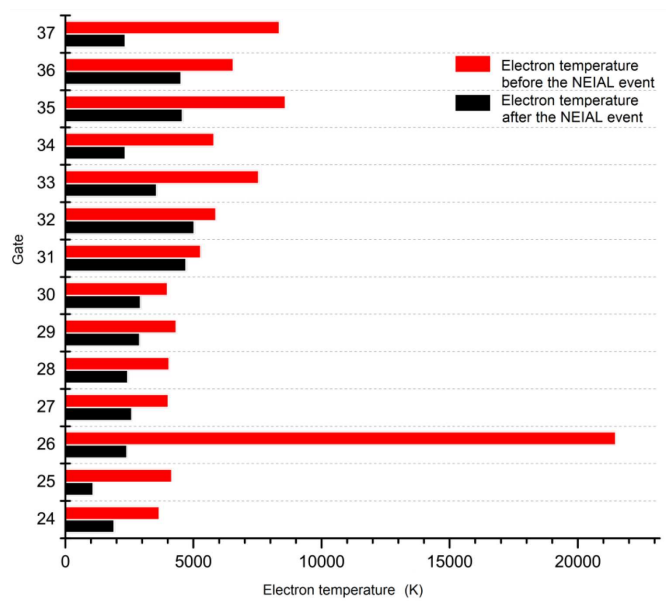

Fig. 4. Histogram of the distribution of the electron temperature on the eve (the red line plot) and after the NEIAL event, for every studied altitude gate. The black bar represents the temperature before the event, while the red bar represents the temperature after the event.

\section{Conclusion}

Our findings give us an argument to support our approach and inductively conclude that the ionospheric plasma processes, manifesting NEIALs, are associated with an increase of plasma electron temperature. Our analysis showed that the electron temperature progressively increased on the eve of the event and progressively decreased after the event. Analysis and the synthesis for the event studied, gave us an estimate for the electron temperature values that exceeded $6600 \mathrm{~K}$ during the event. Our findings support Rietveld et al. [9] who reported that the electron temperatures at high latitude 
ionosphere varied between $4000 \mathrm{~K}$ and $8000 \mathrm{~K}$ at $450 \mathrm{~km}$. The main contribution of this article is the use of an original approach for evaluation the electron temperature fluctuations on the eve and after the NEIAL events observed with the EISCAT Svalbard radar.

\section{Acknowledgments}

EISCAT is an international association supported by research organizations in China (CRIRP), Finland (SA), France (CNRS, till end of 2006), Germany (DFG), Japan (NIPR and STEL), Norway (NFR), Sweden (VR), and the United Kingdom (STFC). The data used in this paper is the property of EISCAT. For accessing the data, we especially thank the UK EISCAT Support Group at the Rutherford Appleton Laboratory.

\section{References}

[1] R.M. Robinson, S.B. Mende, R.R. Vondrak, J.U. Kozyta, A.F. Nagy, J. Geophys. Res. 90, 457 (1985).

[2] E.M. Blixt, T. Grydeland, N. Ivchenko, T. Hagfors, C. La Hoz, B.S. Lanchester, U.P. Løvhaug, T.S. Trondsen, Ann. Geophys. 23, 3 (2005).
[3] M.T. Rietveld, P.N. Collis, J.P. St. Maurice, J. Geophys. Res. 96, 19291 (1991).

[4] J.C. Foster, C. del Pozo, J.P. Groves, K. St. Maurice, Geophys. Res. Lett. 15, 160 (1988).

[5] J.E. Wahlund, H.J. Opgenoorth, F.R.E. Forme, M.A.L. Persson, I. Haggstrom, J. Lilensten, J. Atmos. Terr. Phys. 55, 623 (1993).

[6] F.R.E. Forme, D. Fontaine, J.E. Wahlund, J. Geophys. Res. 100, 14625 (1995).

[7] F.R.E. Forme, D. Fontaine, Ann. Geophys. 17, 182 (1999).

[8] J. Lunde, B. Gustavsson, U.P. Lovhaug, D.A. Lorentzen, Y. Ogawa, Ann. Geophys. 25 1323 (2007)

[9] B. Dalipi, N. Syla, Int. J. Eng. Technol. 13, 42 (2013).

[10] M.S. Lehtinen, A. Huuskonen, J. Pirtilä, Ann. Geophys. 14, 1487 (1996).

[11] B. Dalipi, J. Sci. Res. Rep. 2, 2320 (2013). 